\title{
An Image Multi-scale Feature Recognition Method Based on Image Saliency
}

\author{
Chengzhi Yang \\ Laboratory of Intelligent Information Processing, Suzhou University, Suzhou 234000, Anhui, China \\ Received: July 29, 2020. Revised: March 15, 2021. Accepted: April 5, 2021. Published: April 8, 2021.
}

\begin{abstract}
Image recognition refers to the technology which processes, analyzes and understands images with computer so as to recognize various targets and objects of different patterns. To effectively combine image recognition and intelligent algorithm can enhance the efficiency of image feature analysis, improve the detection accuracy and guarantee real-time detection. In image feature recognition, the following problems exist: the description of accurate object features, object blockage, complex and changeable scenes. Whether these problems can be effectively solved has great significance in improving the stability and robustness of object recognition algorithm. This paper takes image salience as the fundamental framework, and makes in-depth study of the problems of effective object appearance description, multi-feature fusion and multi-feature adaptive combination. Then it proposes an image multi-scale feature recognition method based on image salience and it can better locate the saliency object in the image, and more evenly highlight the salient object and significantly suppress background noises. The experiment results prove that salient region detection algorithm can better stress the entire salient image.
\end{abstract}

Keywords -Multi-scale Feature, Image Recognition, Visual Saliency.

\section{INTRODUCTION}

$\mathrm{I}^{\mathrm{n}}$ $\mathrm{n}$ recent years, obtaining valuable information from images and videos has become a research hotspot in the field of computer vision. Researchers apply computer in the field of image processing to extract valuable information from images, so that people can understand the world more intuitively and accurately. In image processing, computer usually processes all data at the same time, which will consume huge computing resources. However, human visual attention system can selectively notice the regions of interest in complex scenes, and give priority to these regions to obtain valuable information. This processing method is called visual attention mechanism. [1]. Obviously, an image with high definition, contrast and no pollution is the key to guarantee the extraction of follow-up valuable information. Image recognition technology is the process to convert the original image into the digital matrix which can be recognized by machine and then obtain image features through data processing and analysis[2]. Currently, the frequently-used technology is to use digital signal for transmission. It is easy to store and difficult to lose information, which further enhances image recognition rate and ensures image quality. The image recognition technology in machine vision makes it unprecedentedly popular because it can process plenty of information, it can have a highly accurate result and it can perceive sensitively[3]. Visual attention means that human visual system obtains important and compact information from natural scene. As a scene contains too much information, visual attention mechanism can selectively reduce redundant data based on perception and pick out the regions in which humans are interested, also known as salient region. This process is usually called as saliency detection. Human visual system can distinguish the complex scene, quickly find the content of interest, and integrate the information. This is a series of complex body activities, which is completed by human visual attention mechanism. In the field of computer image processing, researchers hope to transform the visual attention mechanism of human visual system into visual saliency algorithm. Researchers use computer algorithms to imitate the process of human visual information processing, efficiently obtain the interesting parts of the image, process the information, and ignore the irrelevant parts. In order to improve the efficiency of image processing, it is very important for computer to detect saliency by simulating human visual attention mechanism, which effectively improves the computer's ability to process visual information [4]. Among image saliency recognition methods, such features as the shape, color and surface texture of the object are affected by the angle of view and the environment of observation and they change under different angles, lights and shelters. Therefore, people determine the matters through the recognition of local features and build feature index; in this way, even change occurs in the viewing angle or the observing environment, they can still match accurately[5] [6].

In the current stage in which artificial intelligence (AI) develops prosperously, people can acquire geometrical information of object and gradually apply image recognition technology in multi-feature recognition; besides, it needs to search a highly-efficient, accurate, real-time and convenient image recognition method in order to describe, store and understand target [7]. The study of digital image processing and recognition started as early as 1965 . Compared with simulation image, digital image is convenient and can be compressed in the transmission, not easy to get distorted in the transmission 
process and convenient to be processed, which has greatly driven the development of image recognition technology. Object recognition mainly refers to the perception and understanding of the objects and the environment in the 3D world and it belongs to high-level computer vision. It is based on digital image processing and recognition and it integrates the research direction of $\mathrm{AI}$ and systematics and its research achievements are widely applied in various industrial and exploration robot. Modern image recognition technology has one defect: poor adaptivity; once the target image is polluted by strong noises or has big flaws, no ideal result can be obtained[8][9].Visual saliency detection computing is to simulate humans' visual attention mechanism through mathematical modeling and computer the degree of importance of the information in the field of view. The multi-feature integration theory of Treisman et al has provided the theoretical foundation for visual salient computing and it divides the visual processing into feature registration and feature integration. In the former phase, the features are detected and encoded parallel and independently and in the latter stage, feature integration and localization are conducted on the object through centrality attention[10]. Inspired by feature integration theory, Kock and Ullman were the first to propose the computing model about visual attention mechanism, which gets features by means of filtering and the salient image through weighted feature map. Itti had come up with the saliency-based visual attention model in 1998 and made further improvements on the theory of this model in Nature in 2001. Itti's saliency model is the most representative and it has become the standard for bottom-up visual attention models [11] [12].

This paper firstly analyzes the characteristics of mathematical morphological processing of image. Then it studies the common features which are frequently used in salient object detection, including: color, texture, shape, brightness and local features. Based on the above research, it has proposed an image multi-scale feature recognition method based on image saliency. Finally, the testing experiment and analysis have proven that the method is effective.

\section{PRINCIPLE OF VISUAL}

Psychological research has found that the image areas which can produce new and different stimuli, strong stimuli and expected stimuli are easy to attract the attention of the observer, which is called visual saliency.

\section{A. Features included in visual saliency}

In recent years, with the digital image and video as the carrier of a large amount of information, in order to understand and analyze the image content efficiently and fully, and obtain the image information, researchers use the computer to simulate the human visual attention mechanism, so as to quickly locate the target object when analyzing and understanding the image, and improve the ability to understand the image. Most human visual neurons are sensitive only to a small area in the middle, called the center. If stimulation is produced in other areas around the center, the stimulation will inhibit the sensitivity of the center to visual neuron stimulation. The structure of the visual neuron makes it sensitive to the mutation of local space, so it is easy to find out those areas which are more contrast to the surrounding areas [13].

In order to make rational and effective use of limited information processing resources, human beings need to choose the specific information in the region when receiving the external information, and give priority to it. The mechanism of human visual attention has a significant inspiration for the development of intelligent science. In image processing, simulating human visual attention mechanism can quickly screen out regions of interest in complex scenes, which are called focus of attention (FOA). The focus of attention is that there is a more prominent difference between the salient object and the background in the image, so as to attract the observer's priority attention. The contrast between an object and its surroundings is very important to determine whether an object can attract attention. Contrast refers to the difference between an area and its surroundings. The difference can be reflected in color, texture or shape. Contrast can be divided into local contrast and global contrast. Local contrast represents the degree of difference between a certain region and its adjacent regions. The greater the difference is, the greater the contrast is; otherwise, the smaller the contrast is. The global contrast represents the difference between an area and the whole scene. The local and global are not absolute. As the neighborhood involved in the local contrast calculation gradually becomes larger, the local contrast will gradually approach the effect of global contrast [14].

Visual saliency includes two aspects: the appearance feature of image and the semantic feature of label. Adding semantic features of image tags is the biggest feature of this method which is different from the traditional methods. The process of salient region extraction method includes training process and testing process, and the specific steps are as follows [15].

(1) The input image is segmented by super pixel. Hyperpixel is the result of pixel clustering, and it can be considered that the pixels in the superpixel are homogeneous.

(2) The appearance features of the image are extracted and the saliency is calculated.

(3) The semantic information of tags is extracted and the semantic features are calculated.

(4) In the training stage, the saliency based on appearance and semantic features based on tags are trained to get the model.

(5) In the test phase, the saliency based on appearance and semantic features based on tags are input into the model in the training phase to get the final saliency map.

\section{B. Visual attention mechanism}

Visual attention mechanism (VA), that is, when facing a scene, people automatically process regions of interest and selectively ignore regions of no interest. These regions of interest are called saliency regions. As the first way for human beings to receive the surrounding environment, vision can quickly screen out the regions of interest when receiving a large amount of data information from the outside world, discard the regions that are not interested in, and give priority to processing 
and analyzing the objects in the image scene, as well as the relationship between the objects and the objects. Feature extraction and feature analysis play a key role in target recognition. Its theoretical basis is that external stimuli are represented by their various features in human long-term memory; in the process of pattern recognition, first of all, we should analyze the features of stimuli, that is, extract the features related to stimuli, and then combine these features with various thorns in long-term memory Once the best match is obtained, the external stimulus is recognized. Therefore, in the target recognition, it is necessary to model the target according to its various characteristics. The establishment of target model needs to solve a major problem, which is the extraction, description and use of various features of the target [16].

In cognitive science, human beings will selectively pay attention to some information and ignore unimportant information for external information. In the acquired complex scenes, they can always quickly select the information they need to pay attention to or are interested in, ignore other valuable information, and give priority to the analysis and processing of important and valuable information, and analyze and simplify the complex scene This mental activity is called visual attention mechanism. Traditional automatic target recognition technology mainly uses the basic features of image, including color and its distribution, texture, object shape and so on. Target recognition algorithm based on image basic features has the characteristics of simple calculation and stable performance, but these features have certain limitations. In order to get closer to human visual understanding ability and improve the accuracy of target recognition, research in recent years has gradually turned to more complex feature recognition methods based on image targets, such as target structure, advanced visual features, etc. Attention mechanism is only a visual mechanism, which does not use mathematics to define attention strictly. At present, visual attention mechanism is widely used in the field of computer, such as learning and extracting local features of images, detecting salient objects, simulating human neurons in neural networks for feature learning and weight distribution. From the perspective of psychophysiology, researchers divide the mechanism of visual attention into two modes: bottom-up and top-down.

(1) Bottom-up visual attention mechanism

Bottom up is only driven by perceptual data, and guides people's viewpoint to the salient areas in the scene; usually, the areas with strong contrast or obvious difference with the surrounding attract bottom-up attention. Using the color, brightness, edge and other features of the image to judge the difference between the target area and its surrounding pixels, and then calculate the significance of the image area. Itti's saliency model is the most representative, which has become the standard of bottom-up visual attention model. For an input image, the model extracts the primary visual features: color, brightness and orientation, and uses center surround operation to generate feature maps reflecting salience measurement at various scales. After these feature maps are merged into the final salience map, the model can be used to generate salience map, In order to get the position of the winner, the winner is selected by using the strategy of "winner return". Bottom-up is not controlled by consciousness. It is mainly driven by data. It detects saliency map through the stimulation or a series of feature information, including a large number of low-level cues, such as texture, color and space, to detect saliency map. The efficiency of processing information is relatively high, which is the main trend of development [17].

(2) Top-down visual attention mechanism

Top-down method is determined by people's "cognitive factors", such as knowledge, expectation and current goal. The saliency of the image area is calculated according to the specific characteristics of the image. This method is based on task driven attention saliency mechanism. It is driven by task experience, and the target saliency area of current image is predicted by knowledge. The top-down feedback mainly relies on the prior information of cerebral cortex, and is dominated by consciousness, which belongs to task driven type. In saliency map detection, manual annotation feature map is required for supervision training, and the information processing efficiency is low. In the model research of simulating top-down visual attention mechanism, Itti proposed a top-down visual attention model which adjusts and controls visual perception in the form of adjusting psychological threshold function. This top-down visual attention model can be divided into three stages: linear filter stage, heterotopic suppression stage and statistical effective decision-making stage. The visual attention model of Task Wizard constructs a structure related to the task of attention position in the scene. Based on task graph, this paper proposes an ontology including real world entities and their relationships, which can be used to calculate the relevance of salient points [18].

\section{Mathematical Morphological Processing}

Mathematical morphology is a new method to be applied in image processing and pattern recognition. Its basic idea is to measure and extract the corresponding shape from the image with the structural elements of a certain form so as to achieve the purpose of image analysis and recognition. The mathematical foundation of and language used in mathematical morphology is set theory. The application of mathematical morphology can simplify image data, preserve their basic shape property and remove irrelevant structures. On the other hand, the algorithms of mathematical morphology have natural parallel structures [19].

Erosion operation is defined as follows

$$
E=B \otimes S=\left\{(x, y) \mid S_{x y} \subseteq B\right\}
$$

$B$ here is the set of all pixels with a value of 1 in the input image, $S$ is the template and $E$ is the set of all pixels with a value of 1 in the output image, $\otimes$ represents for corrosion operation [20]. This equation means that the structural element moves to point $(x, y)$. If it is entirely included in Set $B$, then $(x, y)$ is the element of Set $E$. For all structural elements in the $3 \times 3$ matrix constituted by 1 , if the pixel of point $(x, y)$ of 
the input image is 1 and the 8 pixels in its neighborhood is also 1 , then the pixel of point $(x, y)$ in the output image is 1 ; otherwise, it is 0 . Erosion operation can eliminate the boundary points of the object and it works very well in removing objects.

Dilation operation is defined as

$$
E=B \oplus S=\left\{(x, y) \mid S_{x y} \cap B \neq \varnothing\right\}
$$

Here, $B$ is the set of all points with a value of 1 in the input image and $E$ is the set of all points with a value of 1 in the output image, $\oplus$ represents for dilation operation. This equation means that the structural element moves to point $(x, y)$, if its intersection with Set $B$ is not empty, $(x, y)$ is an element in Set $E$. for all structural elements in the $3 \times 3$ matrix constituted by 1 , if one of point $(x, y)$ and 8 pixels in the neighborhood in the input image is 1 , the pixel of point $(x, y)$ in the output image is 1 ; otherwise, it is 0 . Dilation operation can increase the area of an object and it works excellently in the tiny cavity in the stuffed object. Both erosion and dilation operations can change the size of an object[15].

Opening operation first erodes and then dilates binary image and the definition equation is as follows

$$
E=(B \otimes S) \oplus S
$$

Opening operation can remove tiny objects, and smoothen object boundary without changing its size. Closing operation first dilates and then erodes binary image and its definition equation is

$$
E=(B \oplus S) \otimes S
$$

Closing operation can fill the tiny cavity in the object, connect the adjacent object, and smoothen object boundary without changing its size.

Opening operation is frequently used to remove small (in contrast to structural elements) and bright details while preserving the overall grayscale and big and bright features unchanged. As the erosion operation in the beginning removes the small but bright details while darkening the image, the follow-up dilation process is used to enhance the entire intensity of the image, but it will not re-introduce the details which have been removed [21].

Closing operation is usually used to eliminate small (in contrast to structural elements) but dark details, while keeping unchanged the bright features. Because the initial dilation operation removes dark details and brightens the image, the subsequent erosion process darkens the image, but it will not bring in the details that are removed again.

The region filling algorithm based on dilation, complementation and intersection requires that the boundary points in the region to be filled are 8-connected. Start from point $P$ within the boundary and fill the entire region with 1 (assume the non-boundary elements are 0 ). The filling process is as follows:

$$
X_{k}=\left(X_{k-1} \oplus B\right) \cap A^{c} \quad k=1,2, \ldots K
$$

Where $B$ is symmetrical element. When $k$ iterates to $X_{k}=X_{k-1}$, the algorithm ends and the union set of Set $X_{k}$ and $A$ is the filling result. The union set of every step in the above process and $A^{c}$ has restricted the result within the region in which we are interested (otherwise, dilation will not end until the entire region is filled). So the above process is also called conditional dilation [22].

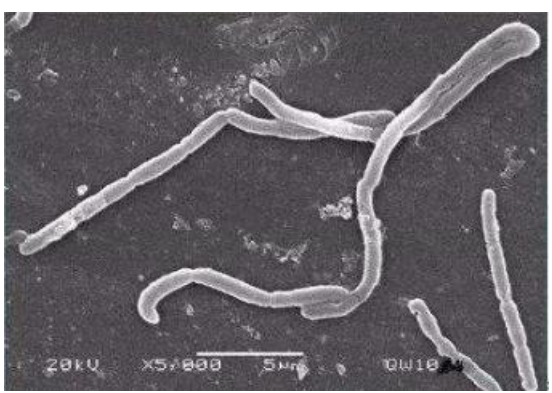

(a) Original image

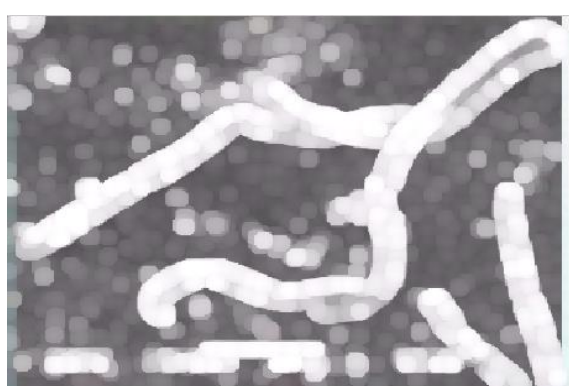

(b) Dilated image

Figure 1. Dilation operation

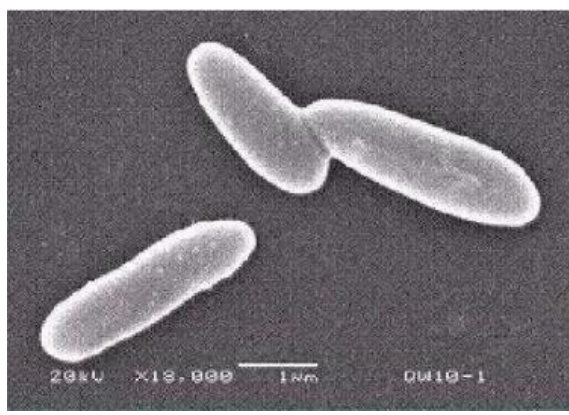

(a) Original image

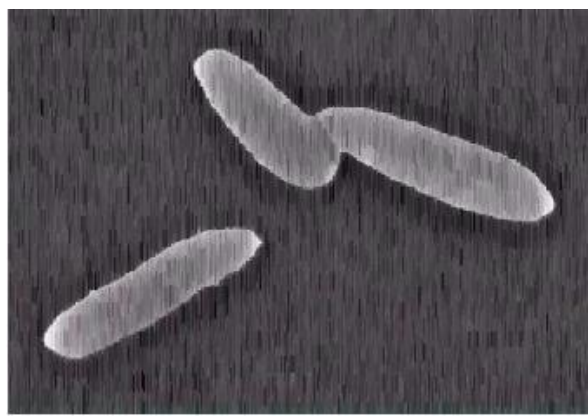




\section{(b) Eroded image}

Fig. 2. Erosion operation

\section{MUlti-SCAlE SALIENCY EXTRACTION}

The salience value of a certain pixel $I_{k}$ in image $I$ is calculated as follows:

$$
\operatorname{SalS}\left(I_{k}\right)=\sum_{\forall I_{k} c I}\left\|I_{k}-I_{i}\right\|
$$

where $k$ represents the $k$ th pixel, the value range of $I_{i}$ is $[0,255]$, i.e. the range of gray value. Expand the equation and get $\operatorname{SalS}\left(I_{k}\right)=\left\|I_{k}-I_{1}\right\|+\left\|I_{k}-I_{2}\right\|+\ldots \ldots+\left\|I_{k}-I_{N}\right\|$ where $N$ represents the number of pixels in the image.

The color value of every pixel $I_{k}$ in the image is known. Assume that $I_{k}=a_{m}$, then the above equation can be further reconstructed:

$$
\begin{aligned}
& \operatorname{Sal} S\left(I_{k}\right)=\left\|a_{m}-a_{0}\right\|+\ldots \ldots+\left\|a_{m}-a_{1}\right\|+\ldots \ldots+ \\
& \operatorname{Sal} S\left(a_{m}\right)=\sum_{n=0}^{255} f_{n}\left\|a_{m}-a_{n}\right\|
\end{aligned}
$$

where $f_{n}$ represents the number of frequency of the nth pixel in the image.

The ultimate goal of region-based saliency detection is to evenly highlight the salient object. In order to enhance the consistency of region-level saliency, the multi-scale saliency extraction in this paper includes the following six steps:

Step1: Calculate the global contrast of a certain pixel in the entire image; in other words, the sum of distances regarding colors between this pixel and all other pixels in the image is the salience value [3]; and then generate the baseline region.

Step 2: By setting the parameters in segmentation algorithm, the original image is segmented into bigger or smaller regions and $\mathrm{M}$ different segmentation scales are formed.

Step 3: With the method of equation (6), get the salience values of the region under all scales.

Step 4: With equation (7), calculate the salience value of every baseline region with the weighted combination of the region in every scale.

Step 5: The salience value of every baseline region is obtained by averaging the salience values under $M$ scales and take the average value of the $M$ salience values of every baseline region obtained in Step 4 as the average salience value of the baseline region.

Step 6: The salience value obtained in Step 5 becomes the final multi-scale salience value.

The above steps can be realized through the equation below. In Step 5, the salience value of the baseline region $r_{p}$ is as follows

$$
S_{1}^{m}\left(r_{p}\right)=\frac{1}{N_{r}} \sum_{\forall q \in r_{p}} S_{\max }^{m}(q)
$$

where $S_{\max }^{m}(q)$ represents the maximum value of the $M$ salience values of a pixel. The salience value of the baseline region $r_{p}$ is as follows:

$$
S_{2}^{m}\left(r_{p}\right)=\frac{1}{M} \sum_{k=1}^{M} S_{2}^{k}\left(r_{p}\right)
$$

where $S_{2}^{k}\left(r_{p}\right)$ represents the salience value of region $r_{p}$ in the $k$ th segmentation scale and this value is obtained through the weighted combination of the salience values in various regions in the $k$ th scale with equation (6). Therefore, in baseline scale, the final salience value of region $r_{p}$ is defined as follows:

$$
S^{m}\left(r_{p}\right)=\exp \left(-\frac{\left\|r_{p}^{c}-I^{c}\right\|}{\sigma_{m}^{2}}\right)\left(\frac{1}{2} S_{1}^{m}\left(r_{p}\right)+\frac{1}{2} S_{2}^{m}\left(r_{p}\right)\right)
$$

where $I^{c}$ represents the image center and parameter $\sigma_{m}$ is used to control the intensity of spatial weight.

\section{TESTING EXPERIMENT AND ANALYSIS}

\section{A. Evaluation Criteria}

For salience object detection tasks, it mainly detects the salient object region which attracts human eyes. The evaluation indicators used in this paper are as follows:

(1) F-measure

As the precision and recall restrict one another, F-measure can be used to comprehensively evaluate them and its definition is shown in equation (11).

$$
F_{\beta}=\frac{\left(1+\beta^{2}\right) \times \text { Precision } \times \text { Recall }}{\beta^{2} \times \text { Precision } \times \text { Recall }}
$$

where $\beta$ is the weight to balance precision and recall. The closer F-measure is to 1 , the better the model has the detection performance.

(2)Area under Curve (AUC)

AUC refers to the area of ROC curve and its value is between 0.5 and 1 . The randomly predicted AUC is 0.5 . The closer it is to 1 , the better the model has the performance.

\section{B. Experimental Analysis}

Subjective evaluation mainly compares the salience image and the true-value image through naked eyes and roughly determines the consistency between them. Objective evaluation 
is to determine the performance of visual saliency detection model by calculating some indicators. Generally, a visual saliency detection model is seen as a binary classifier; so the relevant indicators can be used to determine the model performance. The subjective comparison result of the specific experiment is shown in Fig. 3 and Fig.4 below, the objective comparison result is shown in Tab. 1 below.

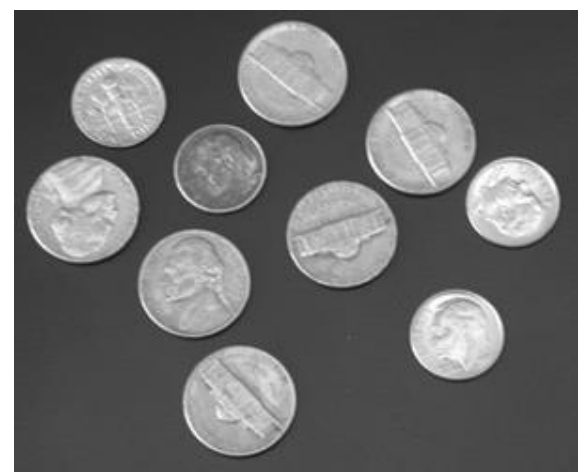

(a) Original image

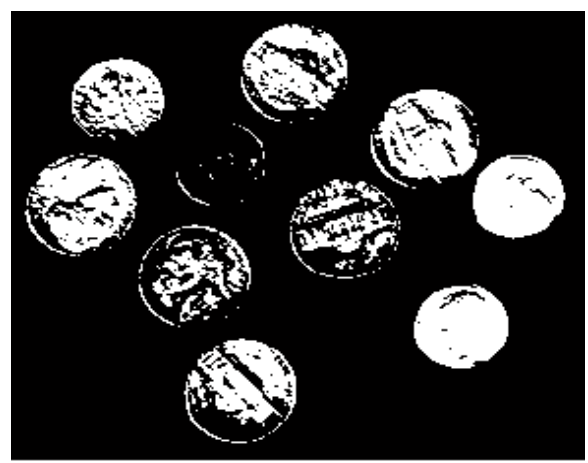

(b) Binary image

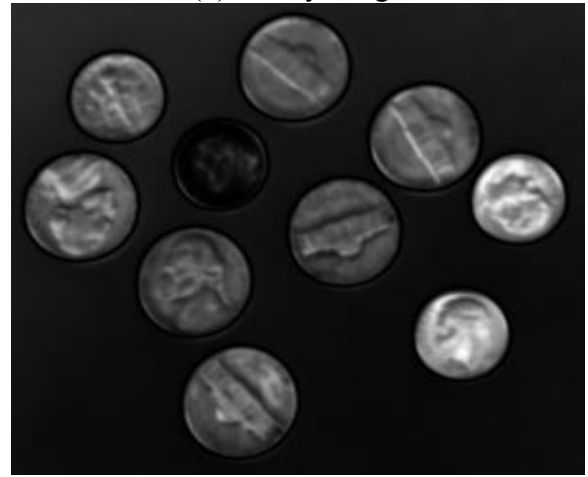

(c) FT algorithm

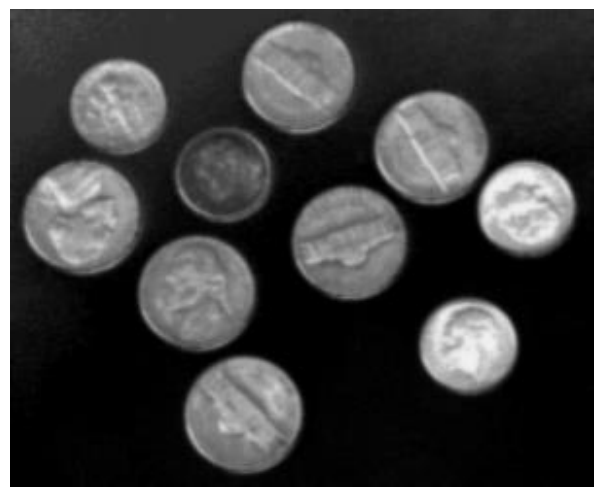

(d) $\mathrm{HC}$ algorithm

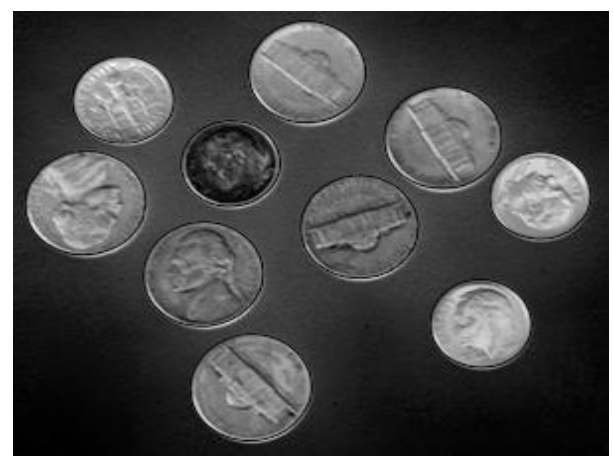

(e) AC algorithm

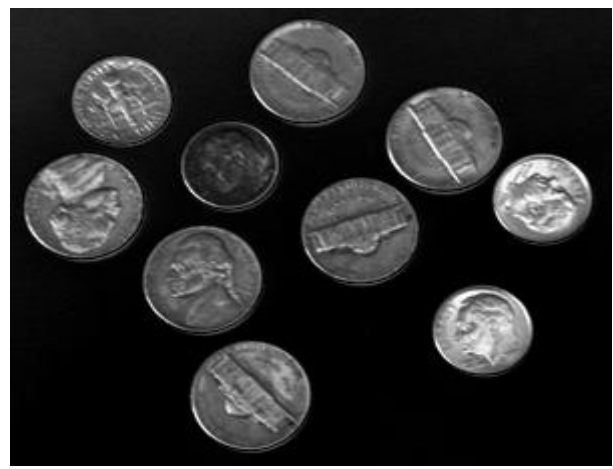

(f) The algorithm in this paper

Fig. 3. The test results of Coins image

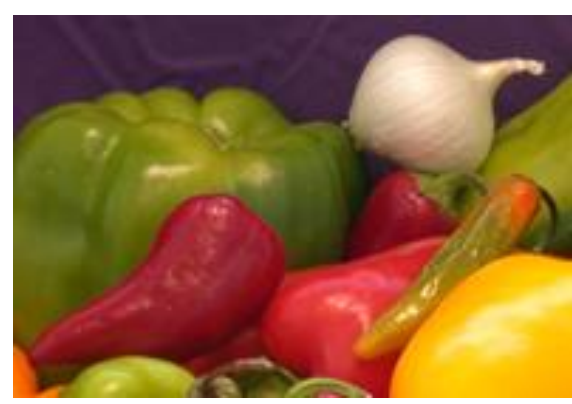

(a) Original image

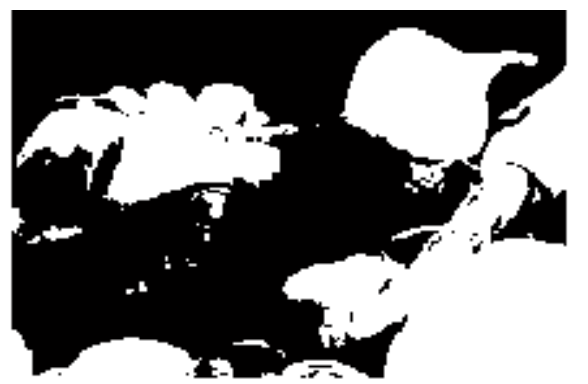

(b) Binary image 


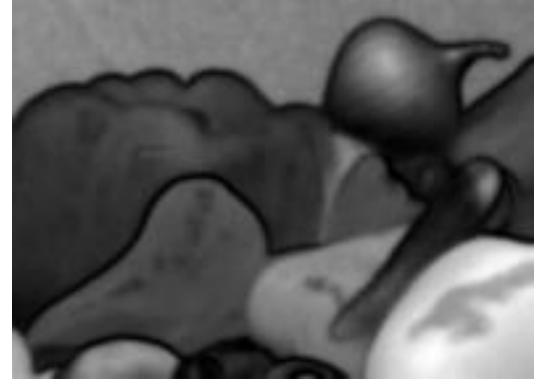

(c) FT algorithm

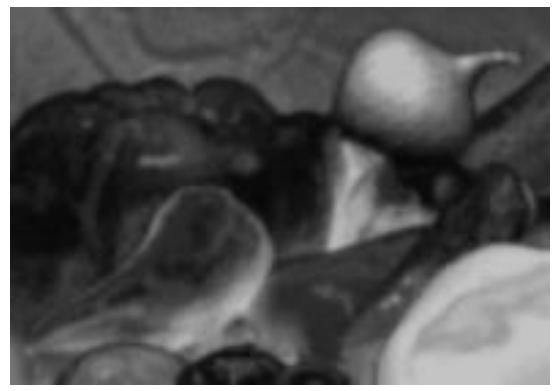

(d)HC algorithm

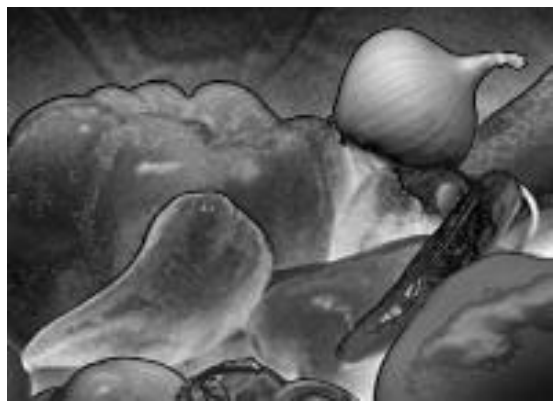

(e) AC algorithm

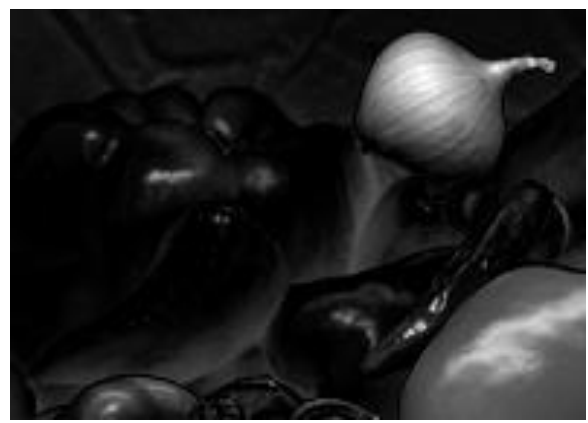

(f) The algorithm in this paper

Fig. 4. The test results of Onion image

Table 1 Results of performance indicator evaluation

\begin{tabular}{|c|c|c|c|c|}
\hline & \multicolumn{2}{|c|}{ Coins image } & \multicolumn{2}{c|}{ Onion image } \\
\cline { 2 - 5 } & FPR & AUC & FPR & AUC \\
\hline FT algorithm & 0.5743 & 0.6972 & 0.6064 & 0.7047 \\
\hline HC algorithm & 0.6281 & 0.6535 & 0.6752 & 0.6755 \\
\hline AC algorithm & 0.6759 & 0.8116 & 0.7008 & 0.7322 \\
\hline $\begin{array}{c}\text { The algorithm in } \\
\text { this paper }\end{array}$ & 0.7367 & 0.8524 & 0.7645 & 0.8019 \\
\hline
\end{tabular}

As shown in the experiment results above, the contrast analysis of global saliency image can separate a large-scale object from its surrounding environment and it is better than the method based on local contrast in which high salience value is generated near the contour. Global consideration can allocate the similar saliency value into the similar image regions and evenly highlight the entire object. The saliency of a region mainly depends on the contrast between it and the adjacent regions and the contrast between it and the far regions is not that important. The algorithm of this paper can better solve the consistent highlight of salience object and the false detection problems and it can more accurately locate the saliency object in microorganism image. Moreover, it suppresses the background surrounding the object and it only highlights the saliency object and it has excellent detection effects on the objects in different types of microscopic image. Through comparison, it can be seen that the algorithm of this paper can more evenly highlight the saliency object and greatly suppress the background noises.

\section{CONCLUSION AND FUTURE WORK}

Image recognition technology-as an indispensable part in computer vision, is also widely applied in the field of various research fields. However, in practice, the image quality obtained in the terminal is not very ideal and the reliability of useful information is severely lowered due to the impact of image acquisition hardware and environment, the image compression encoding, the random noises in transmission channel, and terminal decoding. Therefore, this paper is dedicated to designing an effective multi-scale feature recognition method based on image salience and to address the problems of the existing saliency detections methods, it mainly studies the simulation of human eye's visual attention mechanism and the robustness feature extraction method for image pixels and regions. Meanwhile, this paper also introduces the idea and method of saliency detection into the study of image recognition, which can not only improve the performance of related detection, but also expand the applications of saliency detection. This has theoretical and realistic significance to improve image processing and analysis.

Although image salience have been greatly developed in recent years, there are still many problems to be solved. This paper mainly studies the key problems of saliency detection algorithm, but there are still limitations in this paper, which need further research. The future research should include the following contents

(1) At the same time, it evaluates whether there are significant areas in the image. At present, the proposed saliency detection algorithm can generate saliency map for each input image. But in the actual situation, there are often some scenes that do not have a recognized saliency region. For this situation, this paper will study how to evaluate whether there is a saliency region in the image in the future.

(2) Salient object detection task and visual prediction task are different forms of computer simulation of human visual attention mechanism. There is a strong correlation between the 
two tasks. How to combine the two tasks is a topic worthy of study.

(3) More high-level semantics are fused to complete saliency detection in complex scenes. At present, the performance of this algorithm in some specific scenes needs to be improved, especially when there are many different high-level semantic information in the image. Therefore, this paper will consider how to integrate different high-level semantic features to improve the accuracy of saliency detection in complex scenes in the future.

(4) At present, most salient object detection methods are in the processing of image data, but in the real life scene, video data also occupies a large proportion. At the same time, salient objects may also change with the change of video content, which adds additional challenges to video salient object detection, so it is necessary to design a new salient object detection method for video data. Some other relevant studies can be found in [27], [28], [29].

\section{REFERENCE}

[1] Zhang, Laigang; Wang, Nan; Li, Yibin, Application of Agricultural Remote Sensing Image in Rice Growth Grading Monitoring, Revista De La Facultad De Agronomia De La Universidad Del Zulia, 2019, vol36, no.5, pp.1691-1700.

[2] Jun-Kit Chaw, Musa Mokji, Agricultural Products Recognition System Using Taxonomist's Knowledge as Semantic Attributes, Engineering in Agriculture, Environment and Food, 2016, vol. 9, no. 3, pp.224-234.

[3] Zihao Liu, Fang Cheng, Wei Zhang, A Novel Segmentation Algorithm for Clustered Flexional Agricultural Products Based on Image Analysis", Computers and Electronics in Agriculture, 2016, vol. 126, no. 8, pp.44-54.

[4] J. P. Cobeña Cevallos, J. M. Atiencia Villagomez, I. S. Andryshchenko. "Convolutional Neural Network in the Recognition of Spatial Images of Sugarcane Crops in the Troncal Region of the Coast of Ecuador", Procedia Computer Science, 2019, vol. 150, pp.757-763.

[5] Mikhail Startsev, Michael Do, "360-Aware Saliency Estimation With Conventional Image Saliency Predictors", Signal Processing: Image Communication, 2018, vol. 69, no .11, pp.43-52.

[6] Li, Xiuping; Han, Conghai, Common Posterior Animal Image Recognition System in Activated Sludge", Acta Microscopica, 2019, vol.28, no.5, pp.1279-1288.

[7] YoshinariMorio, Tasuku Inoue, Takaaki Tanaka, Katsusuke Murakami. (2017) "Worker Posture Recognition for Understanding Agricultural Worker Behaviors,Engineering in Agriculture", Environment and Food, 10(3), pp.208-222.

[8] Luo, Yihao; Wang, Ying; Yang, Jie, "Combination of Deep and Handcrafted Features Enabling Automated and Accurate Detections of Early Gastric Cancer Lesions in Histopathology Images", Investigacion Clinica, 2019, vol. 60, no.4, pp.982-988.

[9] Juncheng Ma, Keming Du, Feixiang Zheng, Lingxian Zhang, Zhongfu Sun, A Recognition Method for Cucumber Diseases Using Leaf Symptom Images Based on Deep Convolutional Neural Network", Computers and Electronics in Agriculture, . 2018, vol.154, no.11, pp.18-24.

[10] Zhai Y, Shah M. (2006) "Visual Attention Detection in Video Sequences Using Spatiotemporal Cues", ACM International Conference on Multimedia. pp.815-824.

[11] O. K. Sikha, S. Sachin Kumar, K. P. Soman, Salient Region Detection and Object Segmentation in Color Images Using Dynamic Mode Decomposition, Journal of Computational Science, 2018, 25(3), pp.351-366.

[12] Maryam Karimi, Shadrokh Samavi, Nader Karimi, S. M. Reza Soroushmehr, Kayvan Najarian, Quality Assessment of Retargeted
Images By Salient Region Deformity Analysis", Journal of Visual Communication and Image Representation, 2017, vol.43, no.2, pp.108-118.

[13] Guo Yingchun, Liu Yi, Ma Runxin, Image Saliency Detection Based on Geodesic-like and Boundary Contrast Maps, ETRI Journal, 2019, vol. 41, no. 6, pp. 797-810.

[14] Wang Xu, Sun Zhenhao, Zhang Qiudan, etc, Multi-Exposure Decomposition-Fusion Model for High Dynamic Range Image Saliency Detection, IEEE Transactions on Circuits and Systems for Video Technology, 2020, vol. 30, no. 12, pp. 4409-4420.

[15] Huang Kun, Gao Shenghua, Image Saliency Detection via Multi-scale Iterative CNN, Visual Computer, 2020, vol. 36, no. 7, pp.. 1355-1367

[16] Zhang Xufan, Wang Yong, Chen Zhenxing, etc, Saliency Detection via Image Sparse Representation and Color Features Combination, Multimedia Tools and Applications, 2020, vol., 79no. 31-32, pp. 23147-23159.

[17] Song Shaoyue, Yu Hongkai, Miao Zhenjiang, etc, An Easy-to-hard Learning Strategy for Within-Image Co-Saliency Detection, Neurocomputing, 2019, vol. 358, pp. . 166-176

[18] Song Minghui, Lu Liu, Peng Yuanxi, etc. Infrared \& Visible Images Fusion Based on Redundant Directional Lifting-Based Wavelet and Saliency Detection, Infrared Physics \& Technology, 2019, vol., 101 pp. $.45-55$

[19] Lishuan Hu, Chengming Qi, Qun Wang, Spectral-Spatial Hyperspectral Image Classification Based on Mathematical Morphology Post-Processing, Procedia Computer Science, (2018), vol.129, 93-97.

[20] KavaskarSekar, Nalin Kant Mohanty, Combined Mathematical Morphology and Data Mining Based High Impedance Fault Detection", Energy Procedia, 2017, vol. 117, no. 6, pp.417-423.

[21] C. A. Rishikeshan, H. Ramesh, An Automated Mathematical Morphology Driven Algorithm for Water Body Extraction From Remotely Sensed Images, ISPRS Journal of Photogrammetry and Remote Sensing, . 2018, vol.146, no.12, pp.11-21.

[22] Manuel González-Hidalgo, Sebastia Massanet, Arnau Mir, Daniel Ruiz-Aguilera, Improving Salt and Pepper Noise Removal Using A Fuzzy Mathematical Morphology-Based Filter, Applied Soft Computing, 2018, vol. 63, no.2, pp.167-180.

[23] Tao Lei, Yanning Zhang, Yi Wang, Shigang Liu, Zhe Guo, A Conditionally Invariant Mathematical Morphological Framework for Color Images, Information Sciences Volume, 2017, vol. 387, no.5, pp.34-52.

[24] Nur Aqilah Othman, Hamzah Ahmad, "The Analysis of Covariance Matrix for Kalman Filter based SLAM with Intermittent Measurement", Int. J. of Applied Mathematics, Computational Science and Systems Engineering, pp. 66-70, Volume 1, 2019

[25] Bacha Sawssen, Taouali Okba, Liouane Noureeddine, "A Mammographic Images Classification Technique via the Gaussian Radial Basis Kernel ELM and KPCA, pp. 92-98, Volume 2, 2020

[26] Maria Isabel Garcia-Planas, Sonia Tarragona, "Analysis of behavior of a simple eigenvalue of singular system", Int. J. of Applied Mathematics, Computational Science and Systems Engineering, pp. 41-47, Volume 3, 2021

\section{Creative Commons Attribution License 4.0 (Attribution 4.0 International , CC BY 4.0)}

This article is published under the terms of the Creative Commons Attribution License 4.0

https://creativecommons.org/licenses/by/4.0/deed.en_US 